\title{
Pilot comparative study on the health of vaccinated and unvaccinated 6- to 12-year-old U.S. children
}

\author{
Anthony R Mawson ${ }^{1 *}$, Brian D Ray ${ }^{2}$, Azad R Bhuiyan ${ }^{3}$ and Binu Jacob ${ }^{4}$ \\ ${ }^{1}$ Professor, Department of Epidemiology and Biostatistics, School of Public Health, Jackson State University, Jackson, MS 39213, USA \\ ${ }^{2}$ President, National Home Education Research Institute, PO Box 13939, Salem, OR 97309, USA \\ ${ }^{3}$ Associate Professor, Department of Epidemiology and Biostatistics, School of Public Health, Jackson State University, Jackson, MS 39213, USA \\ ${ }^{4}$ Former graduate student, Department of Epidemiology and Biostatistics School of Public Health, Jackson State University, Jackson, MS 39213, USA
}

\begin{abstract}
Vaccinations have prevented millions of infectious illnesses, hospitalizations and deaths among U.S. children, yet the long-term health outcomes of the vaccination schedule remain uncertain. Studies have been recommended by the U.S. Institute of Medicine to address this question. This study aimed 1) to compare vaccinated and unvaccinated children on a broad range of health outcomes, and 2) to determine whether an association found between vaccination and neurodevelopmental disorders (NDD), if any, remained significant after adjustment for other measured factors. A cross-sectional study of mothers of children educated at home was carried out in collaboration with homeschool organizations in four U.S. states: Florida, Louisiana, Mississippi and Oregon. Mothers were asked to complete an anonymous online questionnaire on their 6- to 12-year-old biological children with respect to pregnancy-related factors, birth history, vaccinations, physician-diagnosed illnesses, medications used, and health services. NDD, a derived diagnostic measure, was defined as having one or more of the following three closely-related diagnoses: a learning disability, Attention Deficient Hyperactivity Disorder, and Autism Spectrum Disorder. A convenience sample of 666 children was obtained, of which 261 (39\%) were unvaccinated. The vaccinated were less likely than the unvaccinated to have been diagnosed with chickenpox and pertussis, but more likely to have been diagnosed with pneumonia, otitis media, allergies and NDD. After adjustment, vaccination, male gender, and preterm birth remained significantly associated with NDD. However, in a final adjusted model with interaction, vaccination but not preterm birth remained associated with NDD, while the interaction of preterm birth and vaccination was associated with a 6.6 -fold increased odds of NDD (95\% CI: 2.8, 15.5). In conclusion, vaccinated homeschool children were found to have a higher rate of allergies and NDD than unvaccinated homeschool children. While vaccination remained significantly associated with NDD after controlling for other factors, preterm birth coupled with vaccination was associated with an apparent synergistic increase in the odds of NDD. Further research involving larger, independent samples and stronger research designs is needed to verify and understand these unexpected findings in order to optimize the impact of vaccines on children's health.
\end{abstract}

Abbreviations: ADHD: Attention Deficit Hyperactivity Disorder; ASD: Autism Spectrum Disorder; AOM: Acute Otitis Media; CDC: Centers for Disease Control and Prevention; CI: Confidence Interval; NDD: Neurodevelopmental Disorders; NHERI: National Home Education Research Institute; OR: Odds Ratio; PCV-7: Pneumococcal Conjugate Vaccine-7; VAERS: Vaccine Adverse Events Reporting System.

\section{Introduction}

Vaccines are among the greatest achievements of biomedical science and one of the most effective public health interventions of the 20th century [1]. Among U.S. children born between 1995 and 2013, vaccination is estimated to have prevented 322 million illnesses, 21 million hospitalizations and 732,000 premature deaths, with overall cost savings of $\$ 1.38$ trillion [2]. About $95 \%$ of U.S. children of kindergarten age receive all of the recommended vaccines as a requirement for school and daycare attendance [3,4], aimed at preventing the occurrence and spread of targeted infectious diseases [5]. Advances in biotechnology are contributing to the development of new vaccines for widespread use [6].

Under the currently recommended pediatric vaccination schedule [7], U.S. children receive up to 48 doses of vaccines for 14 diseases from birth to age six years, a figure that has steadily increased since the 1950s, most notably since the Vaccines for Children program was created in 1994. The Vaccines for Children program began with vaccines targeting nine diseases: diphtheria, tetanus, pertussis, polio,
Haemophilus influenzae type b disease, hepatitis B, measles, mumps, and rubella. Between 1995 and 2013, new vaccines against five other diseases were added for children age 6 and under: varicella, hepatitis A, pneumococcal disease, influenza, and rotavirus vaccine.

Although short-term immunologic and safety testing is performed on vaccines prior to their approval by the U.S. Food and Drug Administration, the long-term effects of individual vaccines and of the vaccination program itself remain unknown [8]. Vaccines are acknowledged to carry risks of severe acute and chronic adverse effects, such as neurological complications and even death [9], but such risks are considered so rare that the vaccination program is believed to be safe and effective for virtually all children [10].

There are very few randomized trials on any existing vaccine recommended for children in terms of morbidity and mortality, in

*Correspondence to: Anthony $\mathrm{R}$ Mawson, Professor, Department of Epidemiology and Biostatistics, School of Public Health, Jackson State University, Jackson, MS 39213, USA, E-mail: Anthony.r.mawson@jsums.edu

Key words: acute diseases, chronic diseases, epidemiology, evaluation, health policy, immunization, neurodevelopmental disorders, vaccination

Received: March 22, 2017; Accepted: April 21, 2017; Published: April 24, 2017 
part because of ethical concerns involving withholding vaccines from children assigned to a control group. One exception, the high-titer measles vaccine, was withdrawn after several randomized trials in west Africa showed that it interacted with the diphtheria-tetanus-pertussis vaccine, resulting in a significant $33 \%$ increase in child mortality [11]. Evidence of safety from observational studies includes a limited number of vaccines, e.g., the measles, mumps and rubella vaccine, and hepatitis $B$ vaccine, but none on the childhood vaccination program itself. Knowledge is limited even for vaccines with a long record of safety and protection against contagious diseases [12]. The safe levels and longterm effects of vaccine ingredients such as adjuvants and preservatives are also unknown [13]. Other concerns include the safety and costeffectiveness of newer vaccines against diseases that are potentially lethal for individuals but have a lesser impact on population health, such as the group B meningococcus vaccine [14].

Knowledge of adverse events following vaccinations is largely based on voluntary reports to the Vaccine Adverse Events Reporting System (VAERS) by physicians and parents. However, the rate of reporting of serious vaccine injuries is estimated to be $<1 \%$ [15]. These considerations led the former Institute of Medicine (now the National Academy of Medicine) in 2005 to recommend the development of a five-year plan for vaccine safety research by the Centers for Disease Control and Prevention (CDC) [16,17]. In its 2011 and 2013 reviews of the adverse effects of vaccines, the Institute of Medicine concluded that few health problems are caused by or associated with vaccines, and found no evidence that the vaccination schedule was unsafe $[18,19]$. Another systematic review, commissioned by the US Agency for Healthcare Research and Quality to identify gaps in evidence on the safety of the childhood vaccination program, concluded that severe adverse events following vaccinations are extremely rare [20]. The Institute of Medicine, however, noted that studies were needed: to compare the health outcomes of vaccinated and unvaccinated children; to examine the long-term cumulative effects of vaccines; the timing of vaccination in relation to the age and condition of the child; the total load or number of vaccines given at one time; the effect of other vaccine ingredients in relation to health outcomes; and the mechanisms of vaccine-associated injury [19].

A complicating factor in evaluating the vaccination program is that vaccines against infectious diseases have complex nonspecific effects on morbidity and mortality that extend beyond prevention of the targeted disease. The existence of such effects poses a challenge to the assumption that individual vaccines affect the immune system independently of each other and have no physiological effect other than protection against the targeted pathogen [21]. The nonspecific effects of some vaccines appear to be beneficial, while in others they appear to increase morbidity and mortality [22,23]. For instance, both the measles and Bacillus Calmette-Guérin vaccine reportedly reduce overall morbidity and mortality [24], whereas the diphtheria-tetanuspertussis [25] and hepatitis B vaccines [26] have the opposite effect. The mechanisms responsible for these nonspecific effects are unknown but may involve inter alia: interactions between vaccines and their ingredients, e.g., whether the vaccines are live or inactivated; the most recently administered vaccine; micronutrient supplements such as vitamin $\mathrm{A}$; the sequence in which vaccines are given; and their possible combined and cumulative effects [21].

A major current controversy is the question of whether vaccination plays a role in neurodevelopmental disorders (NDDs), which broadly include learning disabilities, Attention Deficit Hyperactivity Disorder (ADHD) and Autism Spectrum Disorder (ASD). The controversy has been fueled by the fact that the U.S. is experiencing what has been described as a "silent pandemic" of mostly subclinical developmental neurotoxicity, in which about $15 \%$ of children suffer from a learning disability, sensory deficits, and developmental delays [27,28]. In 1996 the estimated prevalence of ASD was $0.42 \%$. By 2010 it had risen to $1.47 \%$ ( 1 in 68), with 1 in 42 boys and 1 in 189 girls affected [29]. More recently, based on a CDC survey of parents in 2011-2014, $2.24 \%$ of children ( 1 in 45 ) were estimated to have ASD. Rates of other developmental disabilities, however, such as intellectual disability, cerebral palsy, hearing loss, and vision impairments, have declined or remained unchanged [30]. Prevalence rates of Attention Deficit Hyperactivity Disorder (ADHD) have also risen markedly in recent decades [31]. Earlier increases in the prevalence of learning disability have been followed by declining rates in most states, possibly due to changes in diagnostic criteria [32].

It is believed that much of the increase in NDD diagnoses in recent decades has been due to growing awareness of autism and more sensitive screening tools, and hence to greater numbers of children with milder symptoms of autism. But these factors do not account for all of the increase [33]. The geographically widespread increase in ASD and ADHD suggests a role for an environmental factor to which virtually all children are exposed. Agricultural chemicals are a current focus of research [34-37].

A possible contributory role for vaccines in the rise in NDD diagnoses remains unknown because data on the health outcomes of vaccinated and unvaccinated children are lacking. The need for such studies is suggested by the fact that the Vaccine Injury Compensation Program has paid $\$ 3.2$ billion in compensation for vaccine injury since its creation in 1986 [38]. A study of claims compensated by the Vaccine Injury Compensation Program for vaccine-induced encephalopathy and seizure disorder found 83 claims that were acknowledged as being due to brain damage. In all cases it was noted by the Court of Federal Claims, or indicated in settlement agreements, that the children had autism or ASD [39]. On the other hand, numerous epidemiological studies have found no association between receipt of selected vaccines (in particular the combined measles, mumps, and rubella vaccine) and autism $[10,40-45]$, and there is no accepted mechanism by which vaccines could induce autism [46].

A major challenge in comparing vaccinated and unvaccinated children has been to identify an accessible pool of unvaccinated children, since the vast majority of children in the U.S. are vaccinated. Children educated at home ("homeschool children") are suitable for such studies as a higher proportion are unvaccinated compared to public school children [47]. Homeschool families have an approximately equal median income to that of married-couple families nationwide, somewhat more years of formal education, and a higher average family size (just over three children) compared to the national average of just over two children [48-50]. Homeschooling families are slightly overrepresented in the south, about $23 \%$ are nonwhite, and the age distribution of homeschool children in grades $\mathrm{K}-12$ is similar to that of children nationwide [51]. About $3 \%$ of the school-age population was homeschooled in the 2011-2012 school year [52].

The aims of this study were 1) to compare vaccinated and unvaccinated children on a broad range of health outcomes, including acute and chronic conditions, medication and health service utilization, and 2) to determine whether an association found between vaccination and NDDs, if any, remained significant after adjustment for other measured factors. 


\section{Methods}

\section{Study planning}

To implement the study, a partnership was formed with the National Home Education Research Institute (NHERI), an organization that has been involved in educational research on homeschooling for many years and has strong and extensive contacts with the homeschool community throughout the country (www.nheri.org). The study protocol was approved by the Institutional Review Board of Jackson State University.

\section{Study design}

The study was designed as a cross-sectional survey of homeschooling mothers on their vaccinated and unvaccinated biological children ages 6 to 12. As contact information on homeschool families was unavailable, there was no defined population or sampling frame from which a randomized study could be carried out, and from which response rates could be determined. However, the object of our pilot study was not to obtain a representative sample of homeschool children but a convenience sample of unvaccinated children of sufficient size to test for significant differences in outcomes between the groups.

We proceeded by selecting 4 states (Florida, Louisiana, Mississippi, and Oregon) for the survey (Stage 1). NHERI compiled a list of statewide and local homeschool organizations, totaling 84 in Florida, 18 in Louisiana, 12 in Mississippi and 17 in Oregon. Initial contacts were made in June 2012. NHERI contacted the leaders of each statewide organization by email to request their support. A second email was then sent, explaining the study purpose and background, which the leaders were asked to forward to their members (Stage 2). A link was provided to an online questionnaire in which no personally identifying information was requested. With funding limited to 12 months, we sought to obtain as many responses as possible, contacting families only indirectly through homeschool organizations. Biological mothers of children ages 6-12 years were asked to serve as respondents in order to standardize data collection and to include data on pregnancy-related factors and birth history that might relate to the children's current health. The age-range of 6 to 12 years was selected because most recommended vaccinations would have been received by then.

\section{Recruitment and informed consent}

Homeschool leaders were asked to sign Memoranda of Agreement on behalf of their organizations and to provide the number of member families. Non-responders were sent a second notice but few provided the requested information. However, follow-up calls to the leaders suggested that all had contacted their members about the study. Both the letter to families and the survey questions were stated in a neutral way with respect to vaccines. Our letter to parents began:

"Dear Parent, This study concerns a major current health question: namely, whether vaccination is linked in any way to children's long-term health. Vaccination is one of the greatest discoveries in medicine, yet little is known about its long-term impact. The objective of this study is to evaluate the effects of vaccination by comparing vaccinated and unvaccinated children in terms of a number of major health outcomes ..."

Respondents were asked to indicate their consent to participate, to provide their home state and zip code of residence, and to confirm that they had biological children 6 to 12 years of age. The communications company Qualtrics (http://qualtrics.com) hosted the survey website. The questionnaire included only closed-ended questions requiring yes or no responses, with the aim of improving both response and completion rates.
A number of homeschool mothers volunteered to assist NHERI promote the study to their wide circles of homeschool contacts. A number of nationwide organizations also agreed to promote the study in the designated states. The online survey remained open for three months in the summer of 2012. Financial incentives to complete the survey were neither available nor offered.

\section{Definitions and measures}

Vaccination status was classified as unvaccinated (i.e., no previous vaccinations), partially vaccinated (received some but not all recommended vaccinations) and fully vaccinated (received all recommended age-appropriate vaccines), as reported by mothers. These categories were developed on the premise that any long-term effects of vaccines would be more evident in fully-vaccinated than in partially-vaccinated children, and rare or absent in the unvaccinated. Mothers were asked to use their child's vaccination records to indicate the recommended vaccines and doses their child had received. Dates of vaccinations were not requested in order not to overburden respondents and to reduce the likelihood of inaccurate reporting; nor was information requested on adverse events related to vaccines, as this was not our purpose. We also did not ask about dates of diagnoses because chronic illnesses are often gradual in onset and made long after the appearance of symptoms. Since most vaccinations are given before age 6 , vaccination would be expected to precede the recognition and diagnosis of most chronic conditions.

Mothers were asked to indicate on a list of more than 40 acute and chronic illnesses all those for which her child or children had received a diagnosis by a physician. Other questions included the use of health services and procedures, dental check-ups, "sick visits" to physicians, medications used, insertion of ventilation ear tubes, number of days in the hospital, the extent of physical activity (number of hours the child engaged in "vigorous" activities on a typical weekday), number of siblings, family structure (mother and father living in the home, divorced or separated), family income and/or highest level of education of mother or father, and social interaction with children outside the home (i.e., amount of time spent in play or other contact with children outside the household). Questions specifically for the mother included pregnancy-related conditions and birth history, use of medications during pregnancy, and exposure to an adverse environment (defined as living within 1-2 miles of a furniture manufacturing factory, hazardous waste site, or lumber processing factory). NDD, a derived diagnostic category, was defined as having one or more of the following three closely related and overlapping diagnoses: a learning disability, Attention Deficit Hyperactivity Disorder (ADHD) and Autism Spectrum Disorder (ASD) [53].

\section{Statistical methods}

Unadjusted bivariate analyses using chi-square tests were performed initially to test the null hypothesis of no association between vaccination status and health outcomes, i.e., physician-diagnosed acute and chronic illnesses, medications, and the use of health services. In most analyses, partially and fully vaccinated children were grouped together as the "vaccinated" group, with unvaccinated children as the control group. The second aim of the study was to determine whether any association found between vaccination and neurodevelopmental disorders remained significant after controlling for other measured factors. Descriptive statistics on all variables were computed to determine frequencies and percentages for categorical variables and means $( \pm$ SD) for continuous variables. The strength of associations 
between vaccination status and health outcomes were tested using odds ratios (OR) and 95\% Confidence Intervals (CI). Odds ratios describe the strength of the association between two categorical variables measured simultaneously and are appropriate measures of that relationship in a cross-sectional study [54]. Unadjusted and adjusted logistic regression analyses were carried out using SAS (Version 9.3) to determine the factors associated with NDDs.

\section{Results}

\section{Socio-Demographic characteristics of respondents}

The information contained in 415 questionnaires provided data on 666 homeschool children. Table 1 shows the characteristics of the survey respondents. Mothers averaged about 40 years of age, were typically white, college graduates, with household incomes between $\$ 50,000$ to $\$ 100,000$, Christian, and married. The reasons for homeschooling for the majority of respondents (80-86\%) were for a moral environment, better family relationships, or for more contact with their child or children.

The children as a group were similarly mostly white $(88 \%)$, with a slight preponderance of females (52\%), and averaged 9 years of age. With regard to vaccination status, 261 (39\%) were unvaccinated, 208 (31\%) were partially vaccinated, and 197 (30\%) had received all of the recommended vaccinations. All statistical analyses are based on these numbers.

\section{Acute illness}

Vaccinated children $(\mathrm{N}=405)$, combining the partially and fully vaccinated, were significantly less likely than the unvaccinated to have had chickenpox ( $7.9 \%$ vs. $25.3 \%, \mathrm{p}<0.001$; Odds Ratio $=0.26,95 \%$ Confidence Interval: $0.2,0.4)$ and whooping cough (pertussis) $(2.5 \%$ vs. $8.4 \%, \mathrm{p}<0.001$; OR $0.3,95 \%$ CI: $0.1,0.6$ ), and less likely, but not significantly so, to have had rubella $(0.3 \%$ vs. $1.9 \%, \mathrm{p}=0.04$; OR 0.1 , $95 \%$ CI: $0.01,1.1)$. However, the vaccinated were significantly more likely than the unvaccinated to have been diagnosed with otitis media ( $19.8 \%$ vs. $5.8 \%, \mathrm{p}<0.001$; OR $3.8,95 \%$ CI: $2.1,6.6)$ and pneumonia (6.4\% vs. $1.2 \%, \mathrm{p}=0.001$; OR 5.9, 95\% CI: $1.8,19.7)$. No significant differences were seen with regard to hepatitis $A$ or $B$, high fever in the past 6 months, measles, mumps, meningitis (viral or bacterial), influenza, or rotavirus (Table 2).

\section{Chronic illness}

Vaccinated children were significantly more likely than the unvaccinated to have been diagnosed with the following: allergic rhinitis ( $10.4 \%$ vs. $0.4 \%, \mathrm{p}<0.001$; OR $30.1,95 \%$ CI: $4.1,219.3)$, other allergies (22.2\% vs. $6.9 \%, \mathrm{p}<0.001$; OR $3.9,95 \% \mathrm{CI}: 2.3,6.6)$, eczema/ atopic dermatitis ( $9.5 \%$ vs. $3.6 \%, \mathrm{p}=0.035$; OR $2.9,95 \% \mathrm{CI}: 1.4,6.1)$, a learning disability (5.7\% vs. $1.2 \%, \mathrm{p}=0.003$; OR 5.2, 95\% CI: $1.6,17.4)$, $\operatorname{ADHD}(4.7 \%$ vs. $1.0 \%, \mathrm{p}=0.013$; OR $4.2,95 \% \mathrm{CI}: 1.2,14.5)$, ASD $(4.7 \%$ vs. $1.0 \%, \mathrm{p}=0.013$; OR $4.2,95 \% \mathrm{CI}: 1.2,14.5)$, any neurodevelopmental disorder (i.e., learning disability, ADHD or ASD) (10.5\% vs. $3.1 \%, \mathrm{p}$ $<0.001$; OR 3.7, $95 \%$ CI: $1.7,7.9)$ and any chronic illness $(44.0 \%$ vs. $25.0 \%, \mathrm{p}<0.001$; OR $2.4,95 \% \mathrm{CI}: 1.7,3.3)$. No significant differences were observed with regard to cancer, chronic fatigue, conduct disorder, Crohn's disease, depression, Types 1 or 2 diabetes, encephalopathy, epilepsy, hearing loss, high blood pressure, inflammatory bowel disease, juvenile rheumatoid arthritis, obesity, seizures, Tourette's syndrome, or services received under the Individuals with Disabilities Education Act (Table 3).

\section{Partial versus full vaccination}

Partially vaccinated children had an intermediate position between the fully vaccinated and unvaccinated in regard to several but not all health outcomes. For instance, as shown in Table 4, the partially vaccinated had an intermediate (apparently detrimental) position in terms of allergic rhinitis, ADHD, eczema, and learning disability.

\section{Gender differences in chronic illness}

Among the vaccinated (combining partially and fully vaccinated children), boys were more likely than girls to be diagnosed with a chronic condition - significantly so in the case of allergic rhinitis (13.9\% vs. $7.2 \%, \mathrm{p}=0.03$; OR $2.1,95 \%$ CI: $1.1,4.1$ ), ASD (7.7\% vs. $1.9 \%$, $\mathrm{p}=0.006$; OR $4.3,95 \% \mathrm{CI}: 1.4,13.2)$, and any neurodevelopmental disorder (14.4\% vs. 6.7\%, p = 0.01; OR 2.3, 95\% CI: 1.2, 4.6) (Table 5).

\section{Use of medications and health services}

The vaccinated (combining the partially and fully vaccinated) were significantly more likely than the unvaccinated to use medication for allergies (20.0\% vs. $1.2 \%, \mathrm{p}<0.001$; OR $21.5,95 \% \mathrm{CI}: 6.7,68.9)$, to have used antibiotics in the past 12 months ( $30.8 \%$ vs. $15.4 \%, \mathrm{p}<0.001$; OR $2.4,95 \% \mathrm{CI}: 1.6,3.6)$, and to have used fever medications at least once ( $90.7 \%$ vs. $67.8 \%, \mathrm{p}<0.001$; OR $4.6,95 \%$ CI: $3.0,7.1)$. The vaccinated were also more likely to have seen a doctor for a routine checkup in the past 12 months ( $57.6 \%$ vs. $37.2 \%$, p <0.001; OR $2.3,95 \%$ CI: $1.7,3.2$ ), visited a dentist during the past year $(89.4 \%$ vs. $80.5 \%$, p < $<.001$; OR $2.0,95 \%$ CI: 1.3 3.2), visited a doctor or clinic due to illness in the past year $(36.0 \%$ vs. $16.0 \%, \mathrm{p}<0.001$; OR $3.0,95 \%$ CI: $2.0,4.4)$, been fitted with ventilation ear tubes ( $3.0 \%$ vs. $0.4 \%, \mathrm{p}=0.018$; OR $8.0,95 \% \mathrm{CI}: 1.0$, $66.1)$, and spent one or more nights in a hospital (19.8\% vs. $12.3 \%, \mathrm{p}=$ 0.012; OR 1.8, 95\% CI: 1.1, 2.7) (Table 6).

Table 1. Characteristics of the respondents ${ }^{\mathrm{a}}$

\begin{tabular}{|c|c|}
\hline & $\operatorname{Mean}(S D)^{a}$ \\
\hline \multirow[t]{2}{*}{ Age $(n=407)$} & $40.59(6.7)$ \\
\hline & Number $(\%)^{\mathrm{a}}$ \\
\hline \multicolumn{2}{|l|}{ Race } \\
\hline White & $382(92.5 \%)$ \\
\hline Non-White & $21(7.6 \%)$ \\
\hline Total & 413 \\
\hline \multicolumn{2}{|l|}{ Education } \\
\hline High School Graduate or Less & $35(8.5 \%)$ \\
\hline Some College & $114(27.5 \%)$ \\
\hline College Graduate & $187(45.2 \%)$ \\
\hline Post-Graduates & $78(18.5 \%)$ \\
\hline Total & 414 \\
\hline \multicolumn{2}{|l|}{ Total Gross Household Income } \\
\hline$<\$ 49,999$ & $123(30.8 \%)$ \\
\hline$\$ 50,000-100,000$ & $182(45.5 \%)$ \\
\hline$>\$ 100,000$ & $95(23.8 \%)$ \\
\hline Total & 400 \\
\hline \multicolumn{2}{|l|}{ Religious Affiliation } \\
\hline Christianity & $375(91.2 \%)$ \\
\hline Non-Christianity & $36(8.8 \%)$ \\
\hline Total & 411 \\
\hline \multicolumn{2}{|l|}{ Marital Status } \\
\hline Married & $386(93.7 \%)$ \\
\hline Not Married & $26(6.3 \%)$ \\
\hline Total & 412 \\
\hline
\end{tabular}

${ }^{a}$ Missing observations are excluded. 


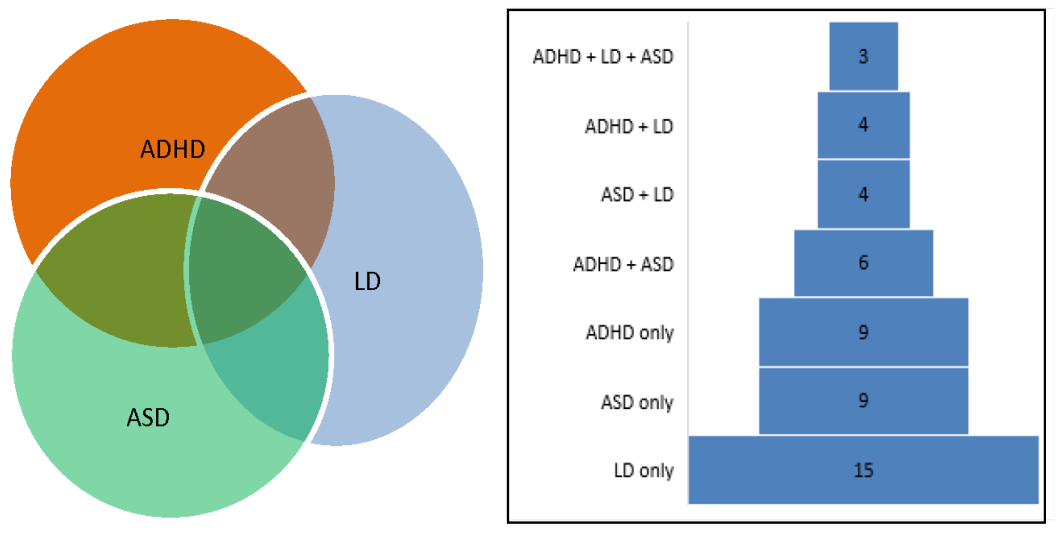

Figure 1. The overlap and distribution of physician-diagnosed neurodevelopmental disorders, based on mothers' reports

Table 2. Vaccination status and health outcomes - Acute Conditions

\begin{tabular}{|c|c|c|c|c|c|c|}
\hline & Vaccinated $(n=405)$ & Unvaccinated $(n=261)$ & Total $(n=666)$ & Chi-square & P-value & Odds Ratio (95\% CI) \\
\hline \multicolumn{7}{|c|}{ Chickenpox } \\
\hline Yes & $32(7.9 \%)$ & $66(25.3 \%)$ & $98(14.7 \%)$ & \multirow{2}{*}{38.229} & \multirow{2}{*}{$<0.001$} & \multirow{2}{*}{$0.26(0.2-0.4)$} \\
\hline No & $373(92.1 \%)$ & $195(74.7 \%)$ & $568(85.3 \%)$ & & & \\
\hline \multicolumn{7}{|c|}{ Otitis media } \\
\hline Yes & $80(19.8 \%)$ & $16(5.8 \%)$ & $96(14.4 \%)$ & \multirow{2}{*}{26.643} & \multirow{2}{*}{$<0.001$} & \multirow{2}{*}{$3.8(2.1-6.6)$} \\
\hline No & $325(80.2 \%)$ & $245(94.2 \%)$ & $507(85.6 \%)$ & & & \\
\hline \multicolumn{7}{|c|}{ Pneumonia } \\
\hline Yes & $26(6.4 \%)$ & $3(1.2 \%)$ & $29(4.4 \%)$ & \multirow{2}{*}{10.585} & \multirow{2}{*}{$<0.001$} & \multirow{2}{*}{$5.9(1.8-19.7)$} \\
\hline No & $379(93.6 \%)$ & $258(98.8 \%)$ & $637(95.6 \%)$ & & & \\
\hline \multicolumn{7}{|c|}{ Whooping cough } \\
\hline Yes & $10(2.5 \%)$ & $22(8.4 \%)$ & $32(4.8 \%)$ & \multirow{2}{*}{12.326} & \multirow{2}{*}{$<0.001$} & \multirow{2}{*}{$0.3(0.1-0.6)$} \\
\hline No & $395(97.5 \%)$ & $239(91.6 \%)$ & $634(95.2 \%)$ & & & \\
\hline \multicolumn{7}{|l|}{ Rubella } \\
\hline Yes & $1(0.3 \%)$ & $5(1.9 \%)$ & $6(0.9 \%)$ & \multirow{2}{*}{4.951} & \multirow{2}{*}{0.037} & \multirow{2}{*}{$0.1(0.01-1.1)$} \\
\hline No & $404(99.6 \%)$ & $256(98.1 \%)$ & $660(99.1 \%)$ & & & \\
\hline
\end{tabular}

Table 3. Vaccination status and health outcomes - Chronic Conditions

\begin{tabular}{|c|c|c|c|c|c|}
\hline Chronic Disease & Vaccinated $(n=405)$ & Unvaccinated $(n=261)$ & Chi-square & P-value & Odds Ratio (95\% CI) \\
\hline \multicolumn{6}{|l|}{ Allergic rhinitis } \\
\hline \multirow[t]{2}{*}{ Yes } & $42(10.4 \%)$ & $1(0.4 \%)$ & \multirow{2}{*}{26.21} & \multirow{2}{*}{$<0.001$} & \multirow{2}{*}{$30.1(4.1-219.3)$} \\
\hline & $363(89.6 \%)$ & $260(99.6 \%)$ & & & \\
\hline \multicolumn{6}{|l|}{ Allergies } \\
\hline Yes & $90(22.2 \%)$ & $18(6.9 \%)$ & \multirow{2}{*}{29.44} & \multirow{2}{*}{$<0.001$} & \multirow{2}{*}{$3.9(2.3-6.6)$} \\
\hline No & $315(77.9 \%)$ & $243(93.1 \%)$ & & & \\
\hline \multicolumn{6}{|l|}{ ADHD } \\
\hline Yes & $19(4.7 \%)$ & $3(1.0 \%)$ & \multirow{2}{*}{6.23} & \multirow{2}{*}{0.013} & \multirow{2}{*}{$4.2(1.2-14.5)$} \\
\hline No & $386(95.3 \%)$ & $258(99.0 \%)$ & & & \\
\hline \multicolumn{6}{|l|}{ ASD } \\
\hline Yes & $19(4.7 \%)$ & $3(1.0 \%)$ & \multirow{2}{*}{6.23} & \multirow{2}{*}{0.013} & \multirow{2}{*}{$4.2(1.2-14.5)$} \\
\hline No & $386(95.3 \%)$ & $258(99.0 \%)$ & & & \\
\hline \multicolumn{6}{|c|}{ Eczema (atopic dermatitis) } \\
\hline Yes & $38(9.5 \%)$ & $9(3.6 \%)$ & \multirow{2}{*}{8.522} & \multirow{2}{*}{0.035} & \multirow{2}{*}{$2.9(1.4-6.1)$} \\
\hline No & $367(90.5 \%)$ & $252(96.4 \%)$ & & & \\
\hline \multicolumn{6}{|l|}{ Learning Disability } \\
\hline Yes & $23(5.7 \%)$ & $3(1.2 \%)$ & \multirow{2}{*}{8.6803} & \multirow{2}{*}{0.003} & \multirow{2}{*}{$5.2(1.6-17.4)$} \\
\hline No & $382(94.3 \%)$ & $258(98.9 \%)$ & & & \\
\hline \multicolumn{6}{|c|}{ Neurodevelopment Disorder } \\
\hline Yes & $42(10.5 \%)$ & $8(3.1 \%)$ & \multirow{2}{*}{12.198} & \multirow{2}{*}{$<0.001$} & \multirow{2}{*}{$3.7(1.7-7.9)$} \\
\hline No & $313(89.5 \%)$ & $253(96.9 \%)$ & & & \\
\hline \multicolumn{6}{|c|}{ Any Chronic Condition } \\
\hline Yes & $178(44.0 \%)$ & $65(24.9 \%)$ & \multirow{2}{*}{24.8456} & \multirow{2}{*}{$<0.001$} & \multirow{2}{*}{$2.4(1.7-3.3)$} \\
\hline No & $227(56.0 \%)$ & $196(75.1 \%)$ & & & \\
\hline
\end{tabular}


Table 4. Partial versus full vaccination and chronic health conditions

\begin{tabular}{|c|c|c|c|c|c|c|}
\hline & Unvaccinated $(n=261)$ & $\begin{array}{l}\text { Partially Vaccinated } \\
\qquad(\mathrm{n}=\mathbf{2 0 8})\end{array}$ & $\begin{array}{l}\text { Fully Vaccinated } \\
(n=197)\end{array}$ & $\begin{array}{c}\text { Total } \\
(n=666)\end{array}$ & Chi-Square & P-value \\
\hline \multicolumn{7}{|c|}{ Chronic Conditions } \\
\hline \multicolumn{7}{|c|}{ Allergic rhinitis } \\
\hline Yes & $1(0.4 \%)$ & $17(8.2 \%)$ & $25(12.7 \%)$ & $43(6.5 \%)$ & \multirow{2}{*}{29.6306} & \multirow{2}{*}{$<0.001$} \\
\hline No & $260(99.6 \%)$ & $191(91.8 \%)$ & $172(87.3 \%)$ & $623(93.5 \%)$ & & \\
\hline \multicolumn{7}{|l|}{ Allergies } \\
\hline Yes & $18(6.9 \%)$ & $47(22.6 \%)$ & $43(21.8 \%)$ & $108(16.2 \%)$ & \multirow{2}{*}{27.4819} & \multirow{2}{*}{$<0.001$} \\
\hline No & $243(93.1 \%)$ & $161(77.4 \%)$ & $154(78.2 \%)$ & $558(83.8 \%)$ & & \\
\hline \multicolumn{7}{|l|}{ ADHD } \\
\hline Yes & $3(1.2 \%)$ & $8(3.9 \%)$ & $11(5.6 \%)$ & $22(3.3 \%)$ & \multirow{2}{*}{7.1900} & \multirow{2}{*}{0.075} \\
\hline No & $258(98.8 \%)$ & $200(96.1 \%)$ & $186(94.4 \%)$ & $644(96.7 \%)$ & & \\
\hline \multicolumn{7}{|l|}{ ASD } \\
\hline Yes & $3(1.2 \%)$ & $11(5.3 \%)$ & $8(4.6 \%)$ & $22(3.3 \%)$ & \multirow{2}{*}{6.7109} & \multirow{2}{*}{0.034} \\
\hline No & $258(98.8 \%)$ & $197(94.7 \%)$ & $189(95.4 \%)$ & $644(96.7 \%)$ & & \\
\hline \multicolumn{7}{|c|}{$\begin{array}{l}\text { Eczema (atopic } \\
\text { dermatitis) }\end{array}$} \\
\hline Yes & $9(3.5 \%)$ & $18(8.7 \%)$ & $20(10.2 \%)$ & $47(7.1 \%)$ & \multirow{2}{*}{8.8683} & \multirow{2}{*}{0.012} \\
\hline No & $252(96.5 \%)$ & $190(91.3 \%)$ & $177(89.8 \%)$ & $619(92.9 \%)$ & & \\
\hline \multicolumn{7}{|c|}{ Learning Disability } \\
\hline Yes & $3(1.2 \%)$ & $11(5.3 \%)$ & $12(6.1 \%)$ & $26(3.9 \%)$ & \multirow{2}{*}{8.8541} & \multirow{2}{*}{0.012} \\
\hline No & $258(98.8 \%)$ & $197(94.7 \%)$ & $185(93.9 \%)$ & $640(96.1 \%)$ & & \\
\hline \multicolumn{7}{|l|}{ NDD } \\
\hline Yes & $8(3.1 \%)$ & $21(10.1 \%)$ & $21(10.7 \%)$ & $50(7.5 \%)$ & \multirow{2}{*}{12.2443} & \multirow{2}{*}{0.002} \\
\hline No & $253(96.9 \%)$ & $187(89.9 \%)$ & $176(89.3 \%)$ & $616(92.5 \%)$ & & \\
\hline \multicolumn{7}{|c|}{ Any Chronic Condition } \\
\hline Yes & $65(24.9 \%)$ & $94(45.2 \%)$ & $84(42.6 \%)$ & $243(36.5 \%)$ & \multirow{2}{*}{25.1301} & \multirow{2}{*}{$<0.001$} \\
\hline No & $196(75.1 \%)$ & $114(54.8 \%)$ & $113(57.4 \%)$ & $423(63.5 \%)$ & & \\
\hline
\end{tabular}

Table 5. Chronic conditions and gender among vaccinated children

\begin{tabular}{|c|c|c|c|c|c|c|}
\hline & $\begin{array}{c}\text { Male } \\
(n=194)\end{array}$ & $\begin{array}{l}\text { Female } \\
(n=209)\end{array}$ & $\begin{array}{c}\text { Total } \\
(n=403)\end{array}$ & Chi-square & P-value & $\begin{array}{l}\text { Odds Ratio } \\
(95 \% \text { CI) }\end{array}$ \\
\hline \multicolumn{7}{|c|}{ Allergic rhinitis } \\
\hline Yes & $27(13.9 \%)$ & $15(7.2 \%)$ & $42(10.4 \%)$ & \multirow{2}{*}{4.8964} & \multirow{2}{*}{0.0269} & \multirow{2}{*}{$2.1(1.1-4.1)$} \\
\hline No & $167(86.1 \%)$ & $194(92.8 \%)$ & $361(90.0 \%)$ & & & \\
\hline \multicolumn{7}{|l|}{ Allergies } \\
\hline Yes & $50(25.8 \%)$ & $40(19.1 \%)$ & $90(22.3 \%)$ & \multirow{2}{*}{2.5531} & \multirow{2}{*}{0.1101} & \multirow{2}{*}{$1.5(0.91-2.4)$} \\
\hline No & $144(74.2 \%)$ & $168(80.9 \%)$ & $313(77.7 \%)$ & & & \\
\hline \multicolumn{7}{|l|}{ ADHD } \\
\hline Yes & $13(6.7 \%)$ & $6(2.9 \%)$ & $19(4.7 \%)$ & \multirow{2}{*}{3.2856} & \multirow{2}{*}{0.0699} & \multirow{2}{*}{$2.4(0.90-6.5)$} \\
\hline No & $181(93.3 \%)$ & $203(97.1 \%)$ & $384(95.3 \%)$ & & & \\
\hline \multicolumn{7}{|l|}{ ASD } \\
\hline Yes & $15(7.7 \%)$ & $4(1.9 \%)$ & $19(4.7 \%)$ & \multirow{2}{*}{7.5810} & \multirow{2}{*}{0.0059} & \multirow{2}{*}{$4.3(1.4-13.2)$} \\
\hline No & $178(92.3 \%)$ & $205(98.1 \%)$ & $384(95.3 \%)$ & & & \\
\hline \multicolumn{7}{|l|}{ Eczema } \\
\hline Yes & $19(9.89 \%)$ & $19(9.1 \%)$ & $38(9.4 \%)$ & \multirow{2}{*}{0.0582} & \multirow{2}{*}{0.8094} & \multirow{2}{*}{$1.1(0.6-2.1)$} \\
\hline No & $175(90.2 \%)$ & $190(90.9 \%)$ & $365(90.6 \%)$ & & & \\
\hline \multicolumn{7}{|c|}{ Learning Disability } \\
\hline Yes & $14(7.2 \%)$ & $9(4.3 \%)$ & $23(5.7 \%)$ & \multirow{2}{*}{1.5835} & \multirow{2}{*}{0.2083} & \multirow{2}{*}{$1.7(0.7-4.1)$} \\
\hline No & $180(92.8 \%)$ & $200(95.7 \%)$ & $380(94.3 \%)$ & & & \\
\hline \multicolumn{7}{|l|}{ NDD } \\
\hline Yes & $28(14.4 \%)$ & $14(6.7 \%)$ & $42(10.4 \%)$ & \multirow{2}{*}{6.4469} & \multirow{2}{*}{0.0111} & \multirow{2}{*}{$2.3(1.2-4.6)$} \\
\hline No & $166(85.6 \%)$ & $195(93.3 \%)$ & $361(89.6 \%)$ & & & \\
\hline \multicolumn{7}{|c|}{ Any Chronic Condition } \\
\hline Yes & $94(48.5 \%)$ & $83(39.7 \%)$ & $177(43.9 \%)$ & \multirow{2}{*}{3.1208} & \multirow{2}{*}{0.0773} & \multirow{2}{*}{$1.4(1.0-2.1)$} \\
\hline No & $100(51.5 \%)$ & $126(60.3 \%)$ & $226(56.1 \%)$ & & & \\
\hline
\end{tabular}

\section{Factors associated with neurodevelopmental disorders}

The second aim of the study focused on a specific health outcome and was designed to determine whether vaccination was associated with neurodevelopmental disorders (NDD) and, if so, whether the association remained significant after adjustment for other measured factors. As noted, because of the relatively small numbers of children with specific diagnoses, NDD was a derived variable combining children with a diagnosis of one or more of ASD, ADHD and a learning disability. The close association and overlap of these diagnoses in the 
Table 6. Vaccination status, medication use and health services utilization

\begin{tabular}{|c|c|c|c|c|c|c|}
\hline & Vaccinated $(n=405)$ & Unvaccinated $(n=261)$ & Total $(n=666)$ & Chi-square & P-value & Odds Ratio $(95 \%$ CI) \\
\hline \multicolumn{7}{|c|}{ Medication Use } \\
\hline \multicolumn{7}{|c|}{ Medication for Allergy } \\
\hline Yes & $81(20.0 \%)$ & $3(1.2 \%)$ & $84(12.6 \%)$ & \multirow{2}{*}{51.170} & \multirow{2}{*}{$<0.001$} & \multirow{2}{*}{$21.5(6.7-68.9)$} \\
\hline No & $324(80.0 \%)$ & $258(98.8 \%)$ & $582(87.4 \%)$ & & & \\
\hline \multicolumn{7}{|c|}{ Used antibiotics in the past 12 months } \\
\hline Yes & $124(30.8 \%)$ & $40(15.4 \%)$ & $164(24.7 \%)$ & \multirow{2}{*}{20.092} & \multirow{2}{*}{$<0.001$} & \multirow{2}{*}{$2.4(1.6-3.6)$} \\
\hline No & $279(69.2 \%)$ & $220(84.6 \%)$ & $499(75.3 \%)$ & & & \\
\hline \multicolumn{7}{|c|}{ Used fever medication $1+$ times } \\
\hline Yes & $350(90.7 \%)$ & $173(67.8 \%)$ & $523(81.6 \%)$ & \multirow{2}{*}{53.288} & \multirow{2}{*}{$<0.001$} & \multirow{2}{*}{$4.6(3.0-7.1)$} \\
\hline No & $36(9.3 \%)$ & $82(32.2 \%)$ & $118(18.4 \%)$ & & & \\
\hline \multicolumn{7}{|c|}{ Using fitted ear drainage tubes } \\
\hline Yes & $12(3.0 \%)$ & $1(0.4 \%)$ & $13(2.0 \%)$ & \multirow[t]{2}{*}{5.592} & \multirow{2}{*}{0.018} & \multirow{2}{*}{$8.0(1.0-66.1)$} \\
\hline No & $389(97.0 \%)$ & $260(99.6 \%)$ & $649(98.0 \%)$ & & & \\
\hline \multicolumn{7}{|c|}{ Used medication for ADHD } \\
\hline Yes & $7(1.7 \%)$ & $3(1.2 \%)$ & $10(1.5 \%)$ & \multirow{2}{*}{0.346} & \multirow{2}{*}{0.556} & \multirow{2}{*}{-} \\
\hline No & $398(98.3 \%)$ & $256(98.8 \%)$ & $654(98.5 \%)$ & & & \\
\hline \multicolumn{7}{|c|}{ Used medication for Seizures } \\
\hline Yes & $4(1.0 \%)$ & $1(0.4 \%)$ & $5(0.8 \%)$ & \multirow{2}{*}{0.769} & \multirow{2}{*}{0.653} & \multirow{2}{*}{-} \\
\hline No & $400(99.0 \%)$ & $258(99.6 \%)$ & $658(99.2)$ & & & \\
\hline \multicolumn{7}{|c|}{ Health Services Utilization } \\
\hline \multicolumn{7}{|c|}{$\begin{array}{c}\text { Emergency Department visit in the past } 12 \\
\text { months }\end{array}$} \\
\hline Yes & $38(9.5 \%)$ & $23(9.0 \%)$ & $61(9.3 \%)$ & \multirow{2}{*}{0.047} & 0828 & 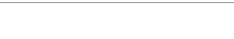 \\
\hline No & $364(90.5 \%)$ & $234(91.0 \%)$ & $598(90.7 \%)$ & & 0.828 & - \\
\hline Sick visit to doctor in & & & & & & \\
\hline Yes & $145(36.0 \%)$ & $41(16.0 \%)$ & $186(28.2 \%)$ & 31096 & $<0001$ & $30(20-44)$ \\
\hline No & $258(64.0 \%)$ & $216(84.0 \%)$ & $474(71.8 \%)$ & 51.090 & -0.001 & $3.0(2.0-4.4)$ \\
\hline Ever spent one or more nig & & & & & & \\
\hline Yes & $80(19.8 \%)$ & $32(12.3 \%)$ & $112(16.8 \%)$ & 6.267 & 0.012 & $18(1.1-27)$ \\
\hline No & $325(80.2 \%)$ & $228(87.7 \%)$ & $553(83.2 \%)$ & & & \\
\hline Seen doctor for checkup it & & & & & & \\
\hline Yes & $233(57.6 \%)$ & $97(37.2 \%)$ & $330(49.6 \%)$ & 26336 & $<0001$ & $23(17-32)$ \\
\hline No & $172(42.4 \%)$ & $164(62.8 \%)$ & $336(50.4 \%)$ & 20.550 & -0.001 & $2.5(1.1-5.2)$ \\
\hline Seen dentist in the pas & & & & & & \\
\hline Yes & $362(89.4 \%)$ & $210(80.5 \%)$ & $572(85.9 \%)$ & 10.424 & $<0.001$ & $2.0(1.3-3.2)$ \\
\hline No & $43(10.6 \%)$ & $51(19.5 \%)$ & $94(14.1 \%)$ & 10.424 & $<0.001$ & $2.0(1.3-3.2)$ \\
\hline
\end{tabular}

study is shown in the figure above (Figure 1). The figure shows that the single largest group of diagnoses was learning disability $(n=15)$ followed by ASD ( $n=9)$, and ADHD ( $n=9)$, with smaller numbers comprising combinations of the three diagnoses.

\section{Unadjusted analysis}

Table 7 shows that the factors associated with NDD in unadjusted logistic regression analyses were: vaccination (OR 3.7, 95\% CI: 1.7, 7.9); male gender (OR 2.1, 95\% CI: 1.1, 3.8); adverse environment, defined as living within 1-2 miles of a furniture manufacturing factory, hazardous waste site, or lumber processing factory (OR 2.9, 95\% CI: 1.1, 7.4); maternal use of antibiotics during pregnancy (OR 2.3, 95\% CI: 1.1, 4.8); and preterm birth (OR 4.9, 95\% CI: 2.4, 10.3). Two factors that almost reached statistical significance were vaccination during pregnancy (OR 2.5, 95\% CI: 1.0, 6.3) and three or more fetal ultrasounds (OR 3.2, 95\% CI: 0.92, 11.5). Factors that were not associated with NDD in this study included mother's education, household income, and religious affiliation; use of acetaminophen, alcohol, and antacids during pregnancy; gestational diabetes; preeclampsia; Rhogham shot during pregnancy; and breastfeeding (data not shown).

\section{Adjusted analysis}

After adjustment for all other significant factors, those that remained significantly associated with NDD were: vaccination (OR 3.1, 95\% CI: 1.4, 6.8); male gender (OR 2.3, 95\% CI: 1.2, 4.3); and preterm birth (OR 5.0, 95\% CI: 2.3, 11.1). The apparently strong association between both vaccination and preterm birth and NDD suggested the possibility of an interaction between these factors.

In a final adjusted model designed to test for this possibility, controlling for the interaction of preterm birth and vaccination, the following factors remained significantly associated with NDD: vaccination (OR 2.5, 95\% CI: 1.1, 5.6), nonwhite race (OR 2.4, 95\% CI: 1.1, 5.4), and male gender (OR 2.3, 95\% CI: 1.2, 4.4). Preterm birth itself, however, was not significantly associated with NDD, whereas the combination (interaction) of preterm birth and vaccination was associated with 6.6-fold increased odds of NDD (95\% CI: 2.8, 15.5) (Table 8).

\section{Discussion}

Following a recommendation of the Institute of Medicine [19] for studies comparing the health outcomes of vaccinated and unvaccinated 
Table 7. Unadjusted analysis of potential risk factors for neurodevelopmental disorders

\begin{tabular}{|c|c|c|c|c|c|c|}
\hline \multicolumn{7}{|c|}{ NDD } \\
\hline Vaccination Status & $\begin{array}{c}\text { Yes } \\
(\mathbf{N}=\mathbf{5 0})\end{array}$ & $\begin{array}{c}\text { No } \\
(\mathrm{N}=616)\end{array}$ & $\begin{array}{c}\text { Total* } \\
(\mathrm{N}=666)\end{array}$ & Chi-Square & P-value & OR $(95 \% \text { CI })^{* *}$ \\
\hline Vaccinated & 42 & 363 & 405 & \multirow{2}{*}{12.198} & \multirow{2}{*}{$<0.001$} & $3.7(1.7-7.9)$ \\
\hline Not Vaccinated & 8 & 253 & 261 & & & Ref \\
\hline \multicolumn{7}{|l|}{ Race } \\
\hline Non-White & 9 & 71 & 80 & \multirow{2}{*}{1.8208} & \multirow{2}{*}{0.177} & $1.7(0.7-3.6)$ \\
\hline White & 41 & 544 & 585 & & & Ref \\
\hline \multicolumn{7}{|l|}{ Child's Gender } \\
\hline Male & 32 & 283 & 315 & \multirow{2}{*}{5.9471} & \multirow{2}{*}{0.015} & $2.1(1.1-3.8)$ \\
\hline Female & 18 & 331 & 349 & & & Ref \\
\hline \multicolumn{7}{|l|}{ Adverse Environment } \\
\hline Yes & 6 & 27 & 33 & \multirow{3}{*}{5.8706} & \multirow{3}{*}{0.053} & $2.9(1.1-7.4)$ \\
\hline No & 40 & 523 & 563 & & & Ref \\
\hline Do not know & 4 & 66 & 70 & & & $0.8(0.3-2.3)$ \\
\hline \multicolumn{7}{|l|}{$\begin{array}{c}\text { Medication during } \\
\text { Pregnancy - Antibiotics }\end{array}$} \\
\hline Yes & 10 & 61 & 71 & \multirow{2}{*}{4.950} & \multirow{2}{*}{0.026} & $2.3(1.1-4.8)$ \\
\hline No & 40 & 555 & 595 & & & Ref \\
\hline \multicolumn{7}{|l|}{$\begin{array}{c}\text { Medication during } \\
\text { Pregnancy -Vaccinated }\end{array}$} \\
\hline Yes & 6 & 32 & 38 & \multirow{2}{*}{3.965} & \multirow{2}{*}{0.057} & $2.5(1.0-6.3)$ \\
\hline No & 44 & 583 & 627 & & & Ref \\
\hline \multicolumn{7}{|l|}{ Preterm birth } \\
\hline Yes & 12 & 37 & 49 & \multirow{2}{*}{22.910} & \multirow{2}{*}{$<0.001$} & $4.9(2.4-10.3)$ \\
\hline No & 38 & 578 & 616 & & & Ref \\
\hline \multicolumn{7}{|l|}{ Ultrasound } \\
\hline None & 3 & 71 & 74 & \multirow{2}{*}{5.898} & \multirow{2}{*}{0.052} & Ref \\
\hline 1-3 times & 30 & 419 & 449 & & & $1.7(0.5-5.7)$ \\
\hline$>3$ times & 17 & 124 & 141 & & & $3.2(0.92-11.5)$ \\
\hline
\end{tabular}

* Numbers may not add to column totals due to missing or incomplete data.

**Note that Odds Ratios are the cross-product ratios of the entries in the 2-by-2 tables, and are an estimate of the relative incidence (or risk) of the outcome associated with the exposure factor.

Table 8. Adjusted logistic regression analyses of risk factors and NDD*

\begin{tabular}{|c|c|c|}
\hline & Adjusted Model (Model 1) & $\begin{array}{c}\text { Adjusted Model with Interaction } \\
\text { (Model 2) }\end{array}$ \\
\hline \multicolumn{3}{|l|}{ Vaccination Status } \\
\hline Vaccinated & $3.1(1.4-6.8)$ & $2.5(1.1-5.6)$ \\
\hline Not Vaccinated & Ref & Ref \\
\hline \multicolumn{3}{|l|}{ Race } \\
\hline Non-White & $2.3(1.0-5.2)$ & $2.4(1.1-5.4)$ \\
\hline White & Ref & Ref \\
\hline \multicolumn{3}{|l|}{ Child's Gender } \\
\hline Male & $2.3(1.2-4.3)$ & $2.3(1.2-4.4)$ \\
\hline Female & Ref & Ref \\
\hline \multicolumn{3}{|l|}{ Preterm birth } \\
\hline Yes & $5.0(2.3-11.1)$ & \multirow[t]{2}{*}{ NS } \\
\hline No & Ref & \\
\hline \multicolumn{3}{|l|}{$\begin{array}{l}\text { Preterm birth } \\
\text { and Vaccination } \\
\text { interaction }\end{array}$} \\
\hline No interaction & \multirow[b]{2}{*}{ Not in the model } & Ref \\
\hline $\begin{array}{l}\text { Preterm and } \\
\text { Vaccinated }\end{array}$ & & $6.6(2.8-15.5)$ \\
\hline
\end{tabular}

*Number of observation read 666 , number of observations used 629. NDD $=47$, Not NDD $=582$

children, this study focused on homeschool children ages 6 to 12 years based on mothers' anonymous reports of pregnancy-related conditions, birth histories, physician-diagnosed illnesses, medications and healthcare use. Respondents were mostly white, married, and college-educated, upper income women who had been contacted and invited to participate in the study by the leaders of their homeschool organizations. Data from the survey were also used to determine whether vaccination was associated specifically with NDDs, a derived diagnostic category combining children with the diagnoses of learning disability, ASD and/or ADHD.

With regard to acute and chronic conditions, vaccinated children were significantly less likely than the unvaccinated to have had chickenpox and pertussis but, contrary to expectation, were significantly more likely to have been diagnosed with otitis media, pneumonia, allergic rhinitis, eczema, and NDD. The vaccinated were also more likely to have used antibiotics, allergy and fever medications; to have been fitted with ventilation ear tubes; visited a doctor for a health issue in the previous year, and been hospitalized. The reason for hospitalization and the age of the child at the time were not determined, but the latter finding appears consistent with a study of 38,801 reports to the VAERS of infants who were hospitalized or had died after receiving vaccinations. The study reported a linear relationship between the number of vaccine doses administered at one time and the rate of hospitalization and death; moreover, the younger the infant at the time of vaccination, the higher was the rate of hospitalization and death [55]. The hospitalization rate increased from $11 \%$ for 2 vaccine doses to $23.5 \%$ for 8 doses $\left(r^{2}=0.91\right)$, while the case fatality rate increased significantly from $3.6 \%$ for those receiving from $1-4$ doses to $5.4 \%$ for those receiving from 5-8 doses.

In support of the possibility that the number of vaccinations received could be implicated in risks of associated chronic illness, a 
comparison of unvaccinated, partially and fully vaccinated children in the present study showed that the partially vaccinated had increased but intermediate odds of chronic disease, between those of unvaccinated and fully vaccinated children, specifically for allergic rhinitis, ADHD, eczema, a learning disability, and NDD as a whole.

The national rates of ADHD and LD are comparable to those of the study. The U.S. rate of ADHD for ages 4-17 (twice the age range of children than the present study), is $11 \%$ [31]. The study rate of ADHD for ages 6 to 12 is 3.3\%, and $4.7 \%$ when only vaccinated children are included. The national LD rate is $5 \%$ [32], and the study data show a rate of $\mathrm{LD}$ of $3.9 \%$ for all groups, and $5.6 \%$ when only vaccinated children are included. However, the ASD prevalence of $2.24 \%$ from a CDC parent survey is lower than the study rate of $3.3 \%$. Vaccinated males were significantly more likely than vaccinated females to have been diagnosed with allergic rhinitis, and NDD. The percentage of vaccinated males with an NDD in this study (14.4\%) is consistent with national findings based on parental responses to survey questions, indicating that $15 \%$ of U.S. children ages 3 to 17 years in the years 2006-2008 had an NDD [28]. Boys are also more likely than girls to be diagnosed with an NDD, and ASD in particular [29].

Vaccination was strongly associated with both otitis media and pneumonia, which are among the most common complications of measles infection $[56,57]$. The odds of otitis media were almost fourfold higher among the vaccinated (OR 3.8, 95\% CI: 2.1, 6.6) and the odds of myringotomy with tube placement were eight-fold higher than those of unvaccinated children (OR 8.0, 95\% CI: 1.0, 66.1). Acute otitis media (AOM) is a very frequent childhood infection, accounting for up to 30 million physician visits each year in the U.S., and the most common reason for prescribing antibiotics for children $[58,59]$. The incidence of AOM peaks at ages 3 to 18 months and $80 \%$ of children have experienced at least one episode by 3 years of age. Rates of AOM have increased in recent decades [60]. Worldwide, the incidence of AOM is $10.9 \%$, with 709 million cases each year, $51 \%$ occurring in children under 5 years of age [61]. Pediatric AOM is a significant concern in terms of healthcare utilization in the U.S., accounting for $\$ 2.88$ billion in annual health care costs [62].

Numerous reports of AOM have been filed with VAERS. A search of VAERS for "Cases where age is under 1 and onset interval is 0 or 1 or 2 or 3 or 4 or 5 or 6 or 7 days and Symptom is otitis media" [63] revealed that 438,573 cases were reported between 1990 and 2011, often with fever and other signs and symptoms of inflammation and central nervous system involvement. One study [64] assessed the nasopharyngeal carriage of $S$. pneumoniae, $H$. influenzae, and $M$. catarrhalis during AOM in fully immunized, partly immunized children with 0 or 1 dose of Pneumococcal Conjugate Vaccine-7 (PCV7), and "historical control" children from the pre-PCV-7 era, and found an increased frequency of $M$. catarrhalis colonization in the vaccinated group compared to the partly immunized and control groups ( $76 \%$ vs. $62 \%$ and $56 \%$, respectively). A high rate of Moraxella catarrhalis colonization is associated with an increased risk of AOM [65].

Successful vaccination against pneumococcal infections can lead to replacement of the latter in the nasopharyngeal niche by nonvaccine pneumococcal serotypes and disease [66]. Vaccination with PCV-7 has a marked effect on the complete microbiota composition of the upper respiratory tract in children, going beyond shifts in the distribution of pneumococcal serotypes and known potential pathogens and resulting in increased anaerobes, gram-positive bacteria and gramnegative bacterial species. PCV-7 administration also correlates highly with the emergence and expansion of oropharyngeal types of species.
These observations have suggested that eradication of vaccine serotype pneumococci can be followed by colonization of other bacterial species in the vacant nasopharyngeal niche, leading to disequilibria of bacterial composition (dysbiosis) and increased risks of otitis media. Long-term monitoring has been recommended as essential for understanding the full implications of vaccination-induced changes in microbiota structure [67].

The second aim of the paper focused on a specific health outcome and sought to determine whether vaccination remained associated with neurodevelopmental disorders (NDD) after controlling for other measured factors. After adjustment, the factors that remained significantly associated with NDD were vaccination, nonwhite race, male gender, and preterm birth. The apparently strong association between both vaccination and preterm birth and NDD suggested the possibility of an interaction between these factors. This was shown in a final adjusted model with interaction (controlling for the interaction of preterm birth with vaccination). In this model, vaccination, nonwhite race and male gender remained associated with NDD, whereas preterm birth itself was no longer associated with NDD. However, preterm birth combined with vaccination was associated with a 6.6-fold increased odds of NDD.

In summary, vaccination, nonwhite race, and male gender were significantly associated with NDD after controlling for other factors. Preterm birth, although significantly associated with NDD in unadjusted and adjusted analyses, was no longer associated with NDD in the final model with interaction. However, preterm birth and vaccination combined was strongly associated with NDD in the final adjusted model with interaction, more than doubling the odds of NDD compared to vaccination alone. Preterm birth has long been known as a major factor for NDD $[68,69]$, but since preterm infants are routinely vaccinated, the separate effects of preterm birth and vaccination have not been examined. The present study suggests that vaccination could be a contributing factor in the pathogenesis of NDD but also that preterm birth by itself may have a lesser or much reduced role in NDD (defined here as ASD, ADHD and/or a learning disability) than currently believed. The findings also suggest that vaccination coupled with preterm birth could increase the odds of NDD beyond that of vaccination alone.

\section{Potential limitations}

We did not set out to test a specific hypothesis about the association between vaccination and health. The aim of the study was to determine whether the health outcomes of vaccinated children differed from those of unvaccinated homeschool children, given that vaccines have nonspecific effects on morbidity and mortality in addition to protecting against targeted pathogens [11]. Comparisons were based on mothers' reports of pregnancy-related factors, birth histories, vaccinations, physician-diagnosed illnesses, medications, and the use of health services. We tested the null hypothesis of no difference in outcomes using chi-square tests, and then used Odds Ratios and 96\% Confidence Intervals to determine the strength and significance of the association.

If the effects of vaccination on health were limited to protection against the targeted pathogens, as is assumed to be the case [21], no difference in outcomes would be expected between the vaccinated and unvaccinated groups except for reduced rates of the targeted infectious diseases. However, in this homogeneous sample of 666 children there were striking differences in diverse health outcomes between the groups. The vaccinated were less likely to have had chickenpox or whooping cough, as expected, but more likely to have been diagnosed with pneumonia and ear infections as well as allergies and NDDs. 
What credence can be given to the findings? This study was not intended to be based on a representative sample of homeschool children but on a convenience sample of sufficient size to test for significant differences in outcomes. Homeschoolers were targeted for the study because their vaccination completion rates are lower than those of children in the general population. In this respect our pilot survey was successful, since data were available on 261 unvaccinated children.

To eliminate opportunities for subjectivity or opinion in the data, only factual information was requested and the questions involved memorable events such as physician-diagnosed diseases in a child. With regard to minimizing potential bias in the information provided by mothers, all communications with the latter emphasized neutrality regarding vaccination and vaccine safety. To minimize recall bias, respondents were asked to use their child's vaccination records. To enhance reliability, closed-ended questions were used and each set of questions had to be completed before proceeding to the next. To enhance validity, parents were asked to report only physiciandiagnosed illnesses.

Mothers' reports could not be validated by clinical records because the survey was designed to be anonymous. However, self-reports about significant events provide a valid proxy for official records when medical records and administrative data are unavailable [70]. Had mothers been asked to provide copies of their children's medical records it would no longer have been an anonymous study and would have resulted in few completed questionnaires. We were advised by homeschool leaders that recruitment efforts would have been unsuccessful had we insisted on obtaining the children's medical records as a requirement for participating in the study.

A further potential limitation is under-ascertainment of disease in unvaccinated children. Could the unvaccinated have artificially reduced rates of illness because they are seen less often by physicians and would therefore have been less likely to be diagnosed with a disease? The vaccinated were indeed more likely to have seen a doctor for a routine checkup in the past 12 months $(57.5 \%$ vs. $37.1 \%, \mathrm{p}<0.001$; OR 2.3 , $95 \%$ CI: $1.7,3.1)$. Such visits usually involve vaccinations, which nonvaccinating families would be expected to refuse. However, fewer visits to physicians would not necessarily mean that unvaccinated children are less likely to be seen by a physician if their condition warranted it. In fact, since unvaccinated children were more likely to be diagnosed with chickenpox and whooping cough, which would have involved a visit to the pediatrician, differences in health outcomes are unlikely to be due to under-ascertainment.

Strengths of the study include the unique design of the study, involving homeschool mothers as respondents, and the relatively large sample of unvaccinated children, which made it possible to compare health outcomes across the spectrum of vaccination coverage. Recruitment of biological mothers as respondents also allowed us to test hypotheses about the role of pregnancy-related factors and birth history as well as vaccination in NDD and other specific conditions. In addition, this was a within-group study of a demographically homogeneous population of mainly white, higher-income and collegeeducated homeschooling families in which the children were all 6-12 years of age. Information was provided anonymously by biological mothers, obviously well-informed about their own children's vaccination status and health, which likely increased the validity of the reports.

\section{Conclusions}

Assessment of the long-term effects of the vaccination schedule on morbidity and mortality has been limited [71]. In this pilot study of vaccinated and unvaccinated homeschool children, reduced odds of chickenpox and whooping cough were found among the vaccinated, as expected, but unexpectedly increased odds were found for many other physician-diagnosed conditions. Although the cross-sectional design of the study limits causal interpretation, the strength and consistency of the findings, the apparent "dose-response" relationship between vaccination status and several forms of chronic illness, and the significant association between vaccination and NDDs all support the possibility that some aspect of the current vaccination program could be contributing to risks of childhood morbidity. Vaccination also remained significantly associated with NDD after controlling for other factors, whereas preterm birth, long considered a major risk factor for NDD, was not associated with NDD after controlling for the interaction between preterm birth and vaccination. In addition, preterm birth coupled with vaccination was associated with an apparent synergistic increase in the odds of NDD above that of vaccination alone. Nevertheless, the study findings should be interpreted with caution. First, additional research is needed to replicate the findings in studies with larger samples and stronger research designs. Second, subject to replication, potentially detrimental factors associated with the vaccination schedule should be identified and addressed and underlying mechanisms better understood. Such studies are essential in order to optimize the impact of vaccination of children's health.

\section{Competing Interests}

The authors declare that they have no financial interests that had any bearing on any aspect of the conduct or conclusions of the study and the submitted manuscript.

\section{Author contributions}

AM designed the study, contributed to data analysis and interpretation, and drafted the paper. BR designed the study, contributed to data collection, and edited the paper. $\mathrm{AB}$ contributed to data analyses and edited the paper. BJ contributed to data analyses and editing. All authors read and approved the final version of the paper.

\section{Funding sources}

This study was supported by grants from Generation Rescue, Inc., and the Children's Medical Safety Research Institute, charitable organizations that support research on children's health and safety. The funders had no role or influence on the design and conduct of the research or the preparation of reports.

\section{Acknowledgments}

The authors thank all those who contributed critical comments, suggestions and financial support for the project. We also thank the collaborating homeschool organizations and especially the mothers who participated in the survey.

\section{Disclaimer}

This study was approved by the Institutional Review Board of Jackson State University and completed prior to Dr. Mawson's tenuretrack appointment at Jackson State University.

\section{References}

1. Centers for Disease Control and Prevention (CDC) (1999) Ten great public health achievements--United States, 1900-1999. MMWR Morb Mortal Wkly Rep 48: 241-243. [Crossref] 
2. Whitney CG, Zhou F, Singleton J, Schuchat A; Centers for Disease Control and Prevention (CDC) (2014) Benefits from immunization during the vaccines for children program era - United States, 1994-2013. MMWR Morb Mortal Wkly Rep 63: 352-355. [Crossref]

3. Centers for Disease Control and Prevention (CDC) (2007) Vaccination coverage among children in kindergarten--United States, 2006-07 school year. MMWR Morb Mortal Wkly Rep 56: 819-821.[Crossref]

4. Centers for Disease Control and Prevention (CDC) (2013) Vaccination coverage among children in kindergarten - United States, 2012-13 school year. MMWR Morb Mortal Wkly Rep 62: 607-612. [Crossref]

5. http://www.cdc.gov/vaccines/vacgen/whatifstop.htm (Accessed 19 June 2016)

6. http://www.hhs.gov/nvpo/vacc_plan/index.html (Accessed 19 June 2015).

7. http://www.cdc.gov/vaccines/schedules/index.html (Accessed 19 June 2016).

8. Ward BJ (2000) Vaccine adverse events in the new millennium: is there reason for concern? Bull World Health Organ 78: 205-215.[Crossref]

9. Sienkiewicz D, Kulak W, Okurowska-Zawada B, Paszko-Pateg G (2012) Neurologic adverse events following vaccination. Prog Health Sci 2:129-141.

10. Pollard AJ (2007) Childhood immunisation: what is the future? Arch Dis Child 92: 426-433. [Crossref]

11. Aaby P, Whittle H, Benn CS (2012) Vaccine programmes must consider their effect on general resistance. $B M J 344$ : e3769. [Crossref]

12. Cunningham AS (2015) Vaccine mandates in the US are doing more harm than good. $B M J$ 351: h4576. [Crossref]

13. Dórea JG. Exposure to mercury and aluminum in early life: developmental vulnerability as a modifying factor in neurologic and immunologic effects. Int J Environ Res Public Health(2015) 12(2):1295-313.

14. Crowcroft NS1, Deeks SL2, Upshur RE2 (2015) Do we need a new approach to making vaccine recommendations? BMJ 350: h308. [Crossref]

15. Kessler DA1 (1993) Introducing MEDWatch. A new approach to reporting medication and device adverse effects and product problems. JAMA 269: 2765-2768. [Crossref]

16. http://www.nap.edu/catalog.php?record_id=11234 (Accessed 19 June 2016).

17. http://www.cdc.gov/vaccinesafety/pdf/iso-finalscientific_agenda-nov-10.pdf(Accessed 19 June 2016).

18. Institute of Medicine (2012) Adverse Effects of Vaccines: Evidence and Causality. The National Academies Press, Washington, DC.

19. Institute of Medicine (2013) The childhood immunization schedule and safety: Stakeholder concerns, scientific evidence, and future studies. The National Academies Press, Washington, DC

20. Maglione MA, Das L, Raaen L, Smith A, Chari R, et al. (2014) Safety of vaccines used for routine immunization of US children: a systematic review. Pediatrics 134:325-337. [Crossref]

21. Siegrist CA (2008) Vaccine Immunology. Vaccines. (5 thedtn). Saunders Elsevier.

22. Benn CS, Netea MG, Selin LK, Aaby P (2013) A small jab - a big effect: nonspecific immunomodulation by vaccines. Trends Immunol 34: 431-439.[Crossref]

23. Jensen KJ, Benn CS, van Crevel R (2016) Unravelling the nature of non-specific effects of vaccines - A challenge for innate immunologists. Semin Immunol 28:377383. [Crossref]

24. Sørup S, Benn CS, Poulsen A, Krause TG, Aaby P, et al. (2014) Live vaccine against measles, mumps, and rubella and the risk of hospital admissions for nontargeted infections. JAMA 311: 826-835.[Crossref]

25. Aaby P, Benn C, Nielsen J, Lisse IM, Rodrigues A, et al. (2012)Testing the hypothesis that diphtheria-tetanus-pertussis vaccine has negative non-specific and sex-differentia effects on child survival in high-mortality countries. BMJ Open 2:e000707. [Crossref]

26. Garly ML1, Jensen H, Martins CL, Balé C, Baldé MA, et al. (2004) Hepatitis B vaccination associated with higher female than male mortality in Guinea-Bissau: an observational study. Pediatr Infect Dis J 23:10861092. [Crossref]

27. Grandjean P, Landrigan PJ (2006) Developmental neurotoxicity of industrial chemicals. Lancet 368: 2167-2178.[Crossref]
28. Boyle CA, Boulet S, Schieve LA, Cohen RA, Blumberg SJ, , et al. (2011) Trends in the prevalence of developmental disabilities in US Children, 1997-2008. Pediatric 127:10341042. [Crossref]

29. Baio J (2014) Prevalence of Autism Spectrum Disorder among children aged 8 years Autism and Developmental Disabilities Monitoring Network, 11 Sites, United States, 2010 Surveillance Summaries. MMWR 63:1-21.

30. Zablotsky B, Black LI, Maenner MJ, Schieve LA, Blumberg SJ (2015) Estimated prevalence of autism and other developmental disabilities following questionnaire changes in the 2014 National Health Interview Survey. Natl Health Stat Report 13:1-20.

31. Visser SN, Danielson ML, Bitsko RH, Holbrook JR, Kogan MD, et al. (2014) Trends in the parent-report of health care provider-diagnosed and medicated attention-deficit hyperactivity disorder: United States, 2003-2011. J Am Acad ChildAdolesc Psychiatry 53:34-46.e2. [Crossref]

32. Cortiella C, Horowitz SH (2014) The State of Learning Disabilities: Facts, Trends and Emerging Issues. National Center for Learning Disabilities, New York:

33. Cornwall W (2015) Autism rates are up, but is it really on the rise? Science Magazine.

34. Landrigan PJ (2010) What causes autism? Exploring the environmental contribution. Curr Opin Pediatr 22: 219-225.[Crossref]

35. Nevison CD (2014) A comparison of temporal trends in United States autism prevalence to trends in suspected environmental factors. Environ Health 13: 73.[Crossref]

36. Shaw CA, Seneff S, Kette SD, Tomljenovic L, Oller JW Jr, et al. (2014) Aluminuminduced entropy in biological systems: implications for neurological disease. $J$ Toxicol 2014: 491316.[Crossref]

37. Sealey LA, Hughes BW, Sriskanda AN1, Guest JR1, Gibson AD1, et al. (2016) Environmental factors in the development of autism spectrum disorders. Environ Int 88: 288-298.[Crossref]

38. http://www.hrsa.gov/vaccinecompensation/data.html (Accessed 20 June 2016).

39. Holland M, Conte L, Krakow R, Colin L (2011)Unanswered questions from the Vaccine Injury Compensation Program: A review of compensated cases of vaccineinduced brain injury. Pace Envtl L Rev 28:480.

40. Doja A, Roberts W (2006) Immunizations and autism: a review of the literature. Can J Neurol Sci33: 341-346.[Crossref]

41. Price CS, Thompson WW, Goodson B, Weintraub ES, Croen LA, et al. (2010) Prenatal and infant exposure to thimerosal from vaccines and immunoglobulins and risk of autism. Pediatrics 126: 656-664.[Crossref]

42. DeStefano F, Price CS, Weintraub ES (2013) Increasing exposure to antibodystimulating proteins and polysaccharides in vaccines is not associated with risk of autism. J Pediatr 163:561-567. [Crossref]

43. McNeil MM, Gee J, Weintraub ES, Belongia EA, Lee GM, et al. (2014) The Vaccine Safety Datalink: successes and challenges monitoring vaccine safety. Vaccine 32: 5390-5398. [Crossref]

44. Taylor LE, Swerdfeger AL, Eslick GD (2014) Vaccines are not associated with autism: an evidence-based meta-analysis of case-control and cohort studies. Vaccine 32: 3623 3629. [Crossref]

45. Jain A, Marshall J, Buikema A, Bancroft T, Kelly JP, et al. (2015) Autism occurrence by MMR vaccine status among US children with older siblings with and without autism. JAMA 313: 1534-1540.[Crossref]

46. Gerber JS, Offit PA (2009) Vaccines and autism: a tale of shifting hypotheses. Clin Infect Dis 48: 456-461.[Crossref]

47. Choi BK, Manning ML (2010) The immunization status of home-schooled children in America. J Pediatr Health Care 24: 42-47.[Crossref]

48. Ray BD (2010) Academic achievement and demographic traits of homeschool students: a nationwide study. J Acad Leadership 8: 1.

49. https://www.census.gov/library/publications/time-series/statistical_abstracts.htm (Accessed 19 August 2016).

50. http://files.eric.ed.gov/fulltext/ED505409.pdf (Accessed 22 August 2016).

51. http://nces.ed.gov/pubs2006/2006042.pdf (Accessed 22 August 2016)

52. http://eric.ed.gov/?id=ED544174 (Accessed 22 August 2016). 
53. Surén P, Bakken IJ, Aase H, Chin R, Gunnes N, et al. (2012) Autism spectrum disorder, ADHD, epilepsy, and cerebral palsy in Norwegian children. Pediatrics 130: e152-158. [Crossref]

54. Zocchetti C, Consonni D, Bertazzi PA (1997) Relationship between prevalence rate ratios and odds ratios in cross-sectional studies. Int J Epidemiol 26: 220-223.[Crossref]

55. Goldman GS, Miller NZ (2012) Relative trends in hospitalizations and mortality among infants by the number of vaccine doses and age, based on the Vaccine Adverse Event Reporting System (VAERS), 1990-2010. Hum ExpToxicol 31:1012-1021. [Crossref]

56. Orenstein WA, Perry RT, Halsey NA (2004)The clinical significance of measles: a review. J Infect Dis 189:S4-S16. [Crossref]

57. CDC (2013) Prevention of measles, rubella, congenital rubella syndrome, and mumps, 2013: Summary Recommendations of the Advisory Committee on Immunization Practices (ACIP). Recommendations and Reports. MMWR 62:1-34.

58. Dhooge IJ (2003) Risk factors for the development of otitis media. Curr Allergy Asthma Rep 3: 321-325. [Crossref]

59. Siegel RM (2010) Acute otitis media guidelines, antibiotic use, and shared medical decision-making. Pediatrics 125:384-386. [Crossref]

60. Casselbrant ML, Mandel EM (2003) Epidemiology. Evidence-based otitis media. BC Decker, Hamilton, ON, Canada. Pp. 147-162.

61. Monasta L1, Ronfani L, Marchetti F, Montico M, VecchiBrumatti L, et al. (2012) Burden of disease caused by otitis media: systematic review and global estimates. PLoS One 7: e36226.[Crossref]

62. Ahmed S1, Shapiro NL, Bhattacharyya N (2014) Incremental health care utilization and costs for acute otitis media in children. Laryngoscope 124: 301-305.[Crossref]
63. http://www.medalerts.org/vaersdb/findfield.php? TA $\mathrm{BLE}=\mathrm{ON} \& \mathrm{GROUP} 1=\mathrm{AGE} \& \mathrm{EVENTS}=\mathrm{ON} \& \mathrm{SYMPTOMS}[]=\mathrm{Otitis}+$ media $+\% 2810033078 \% 29 \&$ NUMDAYS[]=0\&NUMDAYS[] $=1 \&$ NUMDAYS [] $=2 \&$ NUMDAYS [] $=3 \&$ NUMDAYS []$=4 \& N U M D A Y S[]=5 \& N U M-$ DAYS [] $=6 \&$ NUMDAYS [] $=7 \&$ Whic Age $=$ range $\&$ LOWAGE $=0.0 \&$ HIGHAGE $=1.0$ ) (Accessed 25 August, 2016).

64. Revai K, McCormick DP, Patel J, Grady JJ, Saeed K, et al. (2006) Effect of pneumococcal conjugate vaccine on nasopharyngeal bacterial colonization during acute otitis media. Pediatrics 117:1823-1829. [Crossref]

65. Faden H, Harabuchi Y, Hong JJ (1994) Epidemiology of Moraxella catarrhalis in children during the first 2 years of life: relationship to otitis media. J Infect Dis 169: 1312-1317. [Crossref]

66. Weinberger DM, Malley R, Lipsitch M (2011) Serotype replacement in disease after pneumococcal vaccination. Lancet 378:1962-1973. [Crossref]

67. Biesbroek G, Wang X, Keijser BJ, Eijkemans RM, Trzcinski K, et al. (2014) Sevenvalent pneumococcal conjugate vaccine and nasopharyngeal microbiota in healthy children. Emerg Infect Dis 20: 201-210.

68. Goldin RL, Matson JL (2016) Premature birth as a risk factor for autism spectrum disorder. Dev Neurorehabil 19: 203-206.[Crossref]

69. Padilla N, Eklöf E, Mårtensson GE, Bölte S, Lagercrantz H, et al. (2015) Poor brain growth in extremely preterm neonates long before the onset of autism spectrum disorder symptoms. Cereb Cortex 27: 1245-1252. [Crossref]

70. Short ME, Goetzel RZ, Pei X, Tabrizi MJ, Ozminkowski RJ, et al. (2009) How accurate are self-reports? Analysis of self-reported health care utilization and absence when compared with administrative data. J Occup Environ Med 51:786-796. [Crossref]

71. Fisker AB, Hornshøj L, Rodrigues A, Balde I, Fernandes M, et al. (2014) Effects of the introduction of new vaccines in Guinea-Bissau on vaccine coverage, vaccine timeliness, and child survival: an observational study. Lancet Glob Health 2:e478-e487.

Copyright: (C2017 Mawson AR. This is an open-access article distributed under the terms of the Creative Commons Attribution License, which permits unrestricted use, distribution, and reproduction in any medium, provided the original author and source are credited. 